\title{
Evaluation of serum prolidase activity in patients with slow coronary flow
}

\author{
Arif Suner ${ }^{1}$, Abdullah Nurdag ${ }^{2}$, Mustafa Polat ${ }^{1}$, Hakan Kaya $^{1}$, Sedat Koroglư ${ }^{3}$, Gurkan Acar $^{4}$, Hatice Sezen ${ }^{5}$ \\ ${ }^{1}$ Cardiology Department, Faculty of Medicine, Adiyaman University, Adiyaman, Turkey \\ ${ }^{2}$ Cardiology Department, Balikligol State Hospital, Sanliurfa, Turkey \\ ${ }^{3}$ Cardiology Department, Kahramanmaras State Hospital, Kahramanmaras, Turkey \\ ${ }^{4}$ Cardiology Department, Faculty of Medicine, Sutcu Imam University, Kahramanmaras, Turkey \\ ${ }^{5}$ Biochemistry Department, Faculty of Medicine, Harran University, Sanliurfa, Turkey
}

Postep Kardiol Inter 2015; 11, 3 (41): 206-211

DOI: $10.5114 /$ pwki.2015.54015

\begin{abstract}
A bstract
Introduction: Slow coronary flow (SCF) is described as the slow passage of contrast to distal coronaries despite anatomically normal coronary arteries. It has been shown that increased serum prolidase activity (SPA) correlates with collagen turnover. Increased collagen turnover might be associated with the development of atherosclerotic plaques.

Aim: To investigate the relationship between serum prolidase activity and slow coronary flow.

Material and methods: This cross-sectional study included 40 SCF patients (mean age: $55.0 \pm 9.5$ years, 20 females) and 40 controls (mean age: $53.9 \pm 8.2$ years, 21 females) with normal coronary anatomy and normal coronary flow. The Thrombolysis in Myocardial Infarction (TIMI) frame-count (TFC) method was used for SCF diagnosis. Serum prolidase activity was measured spectrophotometrically, and the relevant parameters were compared between the groups.

Results: There were no statistically significant differences between the SCF and control groups in terms of basic demographic, clinical, and laboratory data. However, the SPA was significantly higher in the SCF group compared to the control $(702.7 \pm 13.8$ and $683.9 \pm 13.2$ respectively, $p<0.001)$. Serum prolidase activity was significantly correlated with the mean $\operatorname{TFC}(r=0.463, p<0.001)$. The overall findings of this study support the predictive accuracy of the serum prolidase activity in our cohort, with a statistically significant ROC value of 681.3 .

Conclusions: Our study showed that SPA was increased in SCF patients. The activity of this enzyme was significantly correlated with the mean TFC.
\end{abstract}

Key words: collagen, serum prolidase activity, slow coronary flow.

\section{Introduction}

A recurring problem in clinical practice is determining the cause of chest pain in patients with normal coronary arteries. Detailed angiographic investigations have shown slow passage of contrast in anatomically normal coronaries, known as slow coronary flow (SCF) $[1,2]$. Postulated mechanisms include microvascular, endothelial or vasomotor dysfunction and occlusive disease [2-6].

Types I and III collagen are found in the cardiac matrix and comprise $\sim 80-90 \%$ of the total collagen. As a result of cardiac damage due to myocardial fibrosis, hypertrophy or infarction, changes in the ratios of types I and III collagen lead to structural matrix changes, resulting in myocardial dysfunction $[7,8]$. All of the arteries in the human body contain elastic lamellae, collagen fibrils and smooth muscle cells in the middle layer. So human arteries contain large quantities of collagen [9]. Therefore, any pathological processes affecting the vascular system will affect the collagen cycle. Proline and hydroxyproline constitute $25 \%$ of the amino acids in collagen. Prolidase is a manganese-dependent cytosolic exopeptidase that cleaves iminodipeptides containing a carboxy terminal proline or hydroxyproline, and plays an important role in collagen metabolism, matrix remodeling, and cell growth [10]. Its activity has been documented in plasma, erythrocytes, leukocytes, dermal fibroblasts, and in various organs, such as the kidney, brain, heart, thymus,

\section{Corresponding author:}

Assis. Prof. Arif Suner, Cardiology Department, Faculty of Medicine, Adiyaman University, 02000 Adiyaman, Turkey, phone: +90 05413218581, e-mail: arifsuner@gmail.com

Received: 21.03.2014, accepted: 5.05.2014. 
uterus, liver, small intestine, stomach, spleen, lung, and pancreas [11].

Several studies have demonstrated a relationship between serum prolidase activity (SPA) and cardiovascular diseases, such as the prevalence of coronary artery disease, the occurrence of atrial fibrillation in mitral stenosis, hypertension, and left ventricular hypertrophy in hypertension [12-14].

\section{Aim}

The etiology, diagnosis and management of SCF have been investigated; however, microvascular dysfunction and its connection with prolidase have not been the focus of research to date. The aim of this study was to assess the relationship between SCF and SPA.

\section{Material and methods}

Patients were selected from those attending the clinic with chest pains and who had coronary angiography after non-invasive ischemia tests between July and December 2012. Patients with normal coronary arteries and normal coronary flow $(n=40)$, and patients with normal coronary arteries (three major coronary arteries and their branches $>2 \mathrm{~mm}$ ) but with slow coronary flow in at least one coronary artery $(n=40)$, were included in the study.

The exclusion criteria were as follows: left ventricular systolic dysfunction, hypertrophy, valvular heart disease, pericardial disease, cerebrovascular disease, renal and hepatic dysfunction, hemolytic disease, active infection, autoimmune disease, neoplastic disease, osteoporosis, and any major surgery within the last 3 months. Furthermore, patients with coronary vasospasm, coronary ectasia and any hemodynamic alterations during coronary angiography were also excluded from the study. Additionally, antioxidant drugs or drugs that affected the collagen cycle (angiotensin converting enzyme inhibitor, angiotensin receptor blocker, aldosterone antagonist and statin) were stopped 3 weeks before the start of the study. However, $\beta$-blockers or calcium channel-blockers were prescribed as an antihypertensive therapy, according to the patients' clinical conditions.

The body mass index (BMI) of all patients was calculated. Details on hypertension, hyperlipidemia, diabetes mellitus, family history and smoking were noted. The study was designed according to the tenets of the Helsinki declaration and all patients signed an informed consent form. Complete blood counts, routine biochemical parameters and electrocardiograms were obtained from the patients' hospital records.

\section{Blood sampling protocols}

Serum prolidase activity was measured in both groups. Peripheral venous blood samples were taken from both groups in the fasting state. Blood samples were centrifuged at $3000 \mathrm{rpm}$ for $10 \mathrm{~min}$ to separate the serum, which was stored at $-80^{\circ} \mathrm{C}$ for prolidase analysis.

\section{Prolidase assay}

The serum was diluted 40 -fold with $2.5 \mathrm{mmol} / / \mathrm{Mn}^{2+}$, $40 \mathrm{mmol} / \mathrm{l}$ Trizma HCl buffer ( $\mathrm{pH} \mathrm{8.0)}$ and pre-incubated at $37^{\circ} \mathrm{C}$ for $2 \mathrm{~h}$. The reaction mixture containing $30 \mathrm{mmol} / \mathrm{l}$ gly-pro, $40 \mathrm{mmol} / \mathrm{I}$ Trizma $\mathrm{HCl}$ buffer $(\mathrm{pH} 8.0)$ and $100 \mu \mathrm{l}$ of pre-incubation serum in $1 \mathrm{ml}$ was incubated at $37^{\circ} \mathrm{C}$ for $30 \mathrm{~min}$. Trichloroacetic acid solution $(0.5 \mathrm{ml}, 20 \%)$ was added to stop the incubation reaction. The proline levels in supernatants were assayed by the method proposed by Myara [15], which is a modification of that of Chinard [16]. The intra-assay coefficient of variability (CV) was $3.8 \%$.

\section{Coronary angiography procedure}

Coronary angiography was performed via the femoral artery, using the standard Judkins technique. Coronary angiograms were recorded in the right and left oblique planes using cranial and caudal angulations, with a rate of $30 \mathrm{frames} / \mathrm{s}$ (Allura Xper FD10, Philips Healthcare, Best, The Netherlands). The patients were assessed for the presence of SCF during the coronary angiography, and coronary flow rates were quantified by the TFC method. For objective quantification of the coronary flow, two independent observers performed a blind assessment of the coronary flow in the coronary arteries using the TFC method [17]. Using this method, the number of cine frames, recorded at 30 frames/s, required for the contrast to reach the standard distal coronary landmarks in the left anterior descending (LAD), left circumflex (LCX) and right coronary arteries (RCA) was noted. The predefined distal landmarks were the distal bifurcation for the LAD, commonly referred to as the 'pitchfork' or 'whale's tail', the distal bifurcation of the segment with the longest total distance for the LCX, and the first branch of the posterolateral artery for the RCA. The standard mean values for the normal visualization of coronary arteries are described as $36.2 \pm 2.6$ frames for LAD, $22.2 \pm 4.1$ frames for LCX and $20.4 \pm 3$ frames for RCA. As the LAD coronary artery is usually longer than the other major coronary arteries, the TFC for this vessel is often higher. Therefore, the TFC for LAD is divided by 1.7 to obtain a corrected TFC. The standard corrected mean value for the LAD coronary artery is $21.1 \pm 1.5$ frames. All participants with a corrected TFC greater than two standard deviations from the previously published range for the particular vessel were considered to have SCF [17]. The mean TFC for each patient and control participant was calculated by dividing the sum of the TFC of the corrected LAD, LCX and RCA by three.

\section{Statistical analysis}

SPSS version 15.0 (SPSS Inc., Chicago, IL, USA) was used for the statistical analyses. The one-sample Kolmogorov-Smirnov test was used to determine whether the 
data had a normal distribution. Mean values of continuous variables were compared using the independent two-sample $t$-test. The results were reported as means \pm $\mathrm{SD}$. Categorical variables were compared using the $\chi^{2}$ test and were expressed as counts and percentages. Pearson's correlation coefficients were used to assess the strength of the relationship between the continuous variables. A $p$ value $<0.05$ was considered to indicate significance. The receiver operating characteristics (ROCs) of serum prolidase levels were examined to identify a cut-off value for prediction of SPA.

\section{Results}

The demographic characteristics of both groups, including age, gender, height, weight, body mass index
(BMI), pulse and blood pressure, were similar. Also, coronary artery disease risk factors, such as hypertension, diabetes mellitus, smoking, alcohol use and cholesterol levels, were similar in the two groups $(p>0.05)$, and are shown in Table I.

Serum prolidase activities were significantly higher in the SCF group compared to the control group (702.7 $\pm 13.8 \mathrm{U} / \mathrm{l}, 683.9 \pm 13.2 \mathrm{U} / \mathrm{I}$ respectively, $p<0.001$, Table I, Figure 1). Serum prolidase activity was significantly correlated with the mean TFC $(r=0.463, p<0.001$, Figure 2$)$. The ROC of SPA for prediction of SCF is presented in Figure 3. The area under the curve was 0.82 . The serum prolidase ROC curve analysis showed a sensitivity of $97.5 \%$ (95\% confidence interval (CI): 86.8-99.9\%) and a specificity of $52.5 \%$ ( $95 \% \mathrm{Cl}: 36.1-68.5 \%)$ for the detection of

Table I. Demographic, clinical and angiographic parameters of SCF and control group

\begin{tabular}{|c|c|c|c|}
\hline Variable & SCF group $(n=40)$ & Control group $(n=40)$ & Value of $p$ \\
\hline Gender (male/female) & $20 / 20$ & $19 / 21$ & 0.82 \\
\hline Age [years] & $55.0 \pm 9.5$ & $53.9 \pm 8.2$ & 0.32 \\
\hline Height [cm] & $167 \pm 7$ & $165 \pm 16$ & 0.15 \\
\hline Weight [kg] & $78.5 \pm 9.5$ & $76.4 \pm 11.6$ & 0.38 \\
\hline $\mathrm{BMI}\left[\mathrm{kg} / \mathrm{m}^{2}\right]$ & $28.0 \pm 3.1$ & $28.1 \pm 3.6$ & 0.82 \\
\hline Pulse [beats/min] & $74 \pm 8$ & $74 \pm 10$ & 0.92 \\
\hline Systolic BP [mm Hg] & $128.0 \pm 23.5$ & $130.1 \pm 29.2$ & 0.10 \\
\hline Diastolic BP [mm Hg] & $75.9 \pm 13.9$ & $74.4 \pm 14.9$ & 0.38 \\
\hline Hypertension, $n$ (\%) & $18(45)$ & $18(45)$ & 1.00 \\
\hline Diabetes mellitus, $n(\%)$ & $8(20)$ & $9(22.5)$ & 0.79 \\
\hline Hyperlipidemia, $n$ (\%) & $12(30)$ & $11(27.5)$ & 0.81 \\
\hline Smoking, $n(\%)$ & $9(22.5)$ & $8(20)$ & 0.79 \\
\hline Alcohol, $n(\%)$ & $9(22.5)$ & $4(10)$ & 0.13 \\
\hline CAD family history, $n(\%)$ & $14(35)$ & $13(32.5)$ & 0.81 \\
\hline Total cholesterol [mg/dl] & $194.5 \pm 39.7$ & $199.1 \pm 47.4$ & 0.23 \\
\hline LDL-cholesterol [mg/dl] & $129.2 \pm 39.7$ & $127.1 \pm 37.2$ & 0.79 \\
\hline HDL-cholesterol [mg/dl] & $39.6 \pm 10.1$ & $39 \pm 11.8$ & 0.91 \\
\hline Triglyceride [mg/dl] & $156.4 \pm 74.5$ & $160.4 \pm 97.6$ & 0.72 \\
\hline Urea [mg/dl] & $34 \pm 8$ & $35 \pm 11$ & 0.68 \\
\hline Creatinine $[\mathrm{mg} / \mathrm{dl}]$ & $0.9 \pm 0.3$ & $0.9 \pm 0.3$ & 0.96 \\
\hline Aspirin use, $n(\%)$ & 22 & 15 & 0.11 \\
\hline$\beta$-Blocker, $n$ (\%) & 8 & 11 & 0.43 \\
\hline Calcium channel-blocker, $n$ (\%) & 10 & 7 & 0.41 \\
\hline \multicolumn{4}{|l|}{ TFC (frame/s): } \\
\hline$\overline{L A D}$ & $61.7 \pm 20.5$ & $29.0 \pm 5.8$ & $<0.001$ \\
\hline$\overline{C X}$ & $36.2 \pm 10.6$ & $21.3 \pm 3.5$ & $<0.001$ \\
\hline RCA & $39.7 \pm 12.6$ & $20.0 \pm 3.8$ & $<0.001$ \\
\hline Mean TFC (frame/s) & $45.8 \pm 8.8$ & $24.4 \pm 2.9$ & $<0.001$ \\
\hline $\mathrm{SPA}[\mathrm{U} / \mathrm{I}]$ & $702.7 \pm 13.8$ & $683.9 \pm 13.2$ & $<0.001$ \\
\hline
\end{tabular}

Data presented as mean $\pm S D$, SCF-slow coronary flow, NS-non-significant, BMI-body mass index, CAD-coronary artery disease, $L D L$ - low-density lipoprotein, $H D L$ - high-density lipoprotein, TFC - TIMI frame count, LAD - left anterior descending artery, CX - circumflex artery, RCA - right coronary artery, SPA - serum prolidase activity. 


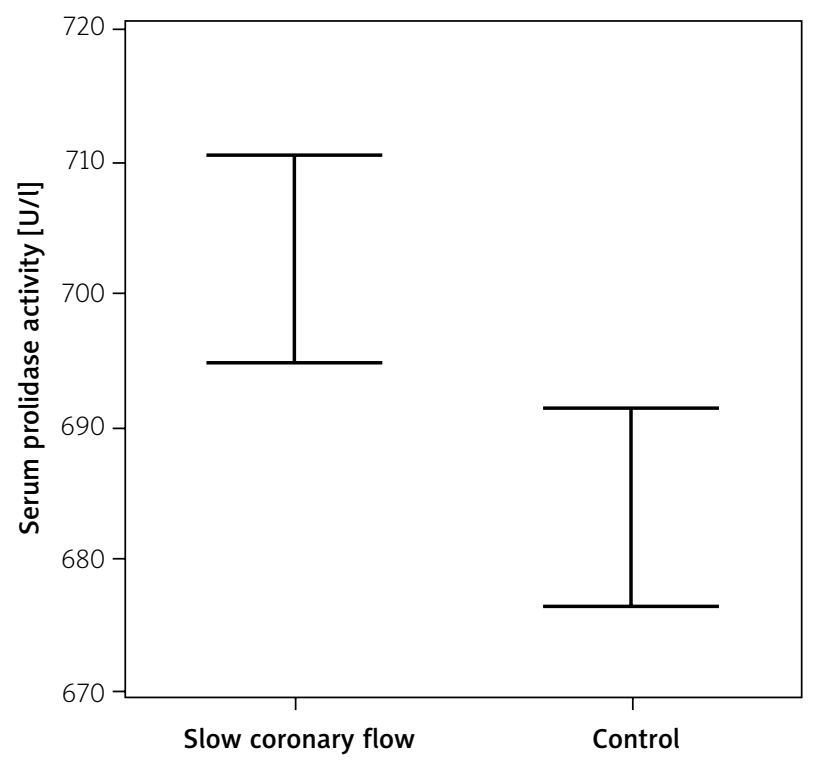

Figure 1. Serum prolidase activity in patients with and without slow coronary flow

SCF, using a serum prolidase activity of $681.3 \mathrm{U} / \mathrm{l}$ as the cut-off.

\section{Discussion}

This study showed that SPA increased in patients with SCF when compared to the controls. The activity of this enzyme was significantly and positively correlated with the mean TFC.

In recent years, studies have found that intimal thickening, diffuse calcification, and atheroma plaques did not cause luminal irregularity in the majority of patients with SCF [3]. It was suggested that oxygen-hemoglobin discordance, and vasomotor and endothelial dysfunction might be responsible for SCF pathophysiology [2-6]. For this reason, the disease was termed 'microvascular angina' because of the possible microvascular involvement [18, 19]. In these studies, the prevalence of recurrent ischemia and myocardial infarction was higher in SCF patients compared to healthy individuals during the long-term follow-up. Despite this, the causes of chest pain and myocardial ischemia remain an ongoing issue for these patients [19, 20].

The cardiac matrix contains mainly types I and III collagen. Proline and hydroxyproline constitute $25 \%$ of the amino acids in collagen [7]. Prolidase hydrolyses proline and hydroxyproline-containing dipeptides intracellularly; proline re-enters the protein cycle and hydroxyproline is excreted in the urine $[7,8]$. Elevated prolidase activity in fibroblasts is associated with increased lysosomal enzymes and fibroblast locomotion, indicating increased collagen turnover [21]. Arterial walls contain mostly collagen [9]; therefore, conditions that affect the arterial system also affect collagen turnover.

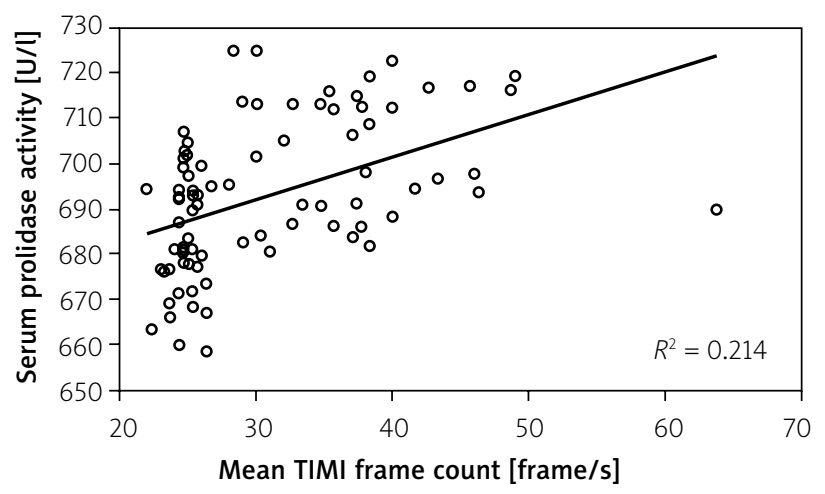

Figure 2. Significant positive correlation between corrected mean TIMI frame count and serum prolidase activity $(r=0.463, p<0.001)$

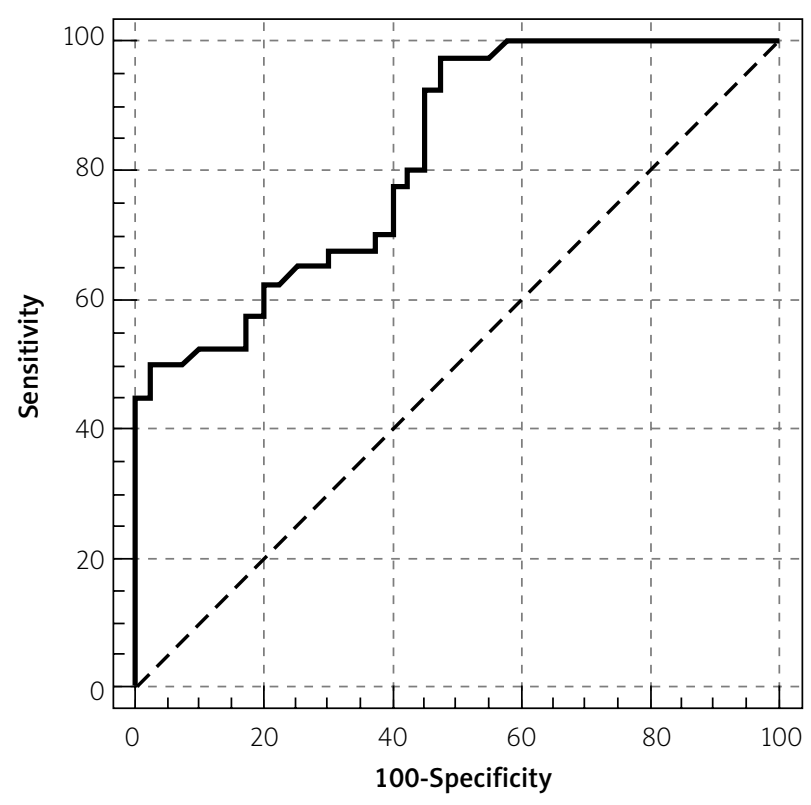

Figure 3. Receiver-operating characteristic (ROC) curve calculation for the serum prolidase activity in slow coronary flow

Serum prolidase activity might also be an indicator of bone turnover [22]. A low level of SPA was reported to be related to osteoporosis [23] and osteoarthritis [24] due to reduced bone turnover. In patients with uremia, low SPA was suggested as a contributory factor to the imino acidopathy [25].

Cardiac damage due to myocardial fibrosis, hypertrophy, and infarction causes changes in the ratio of types I and III collagen, which lead to alterations in the matrix structure and impairment of myocardial function [7]. Serum prolidase activity is elevated in hypertensive patients and is related to increased collagen turnover [14]. The reported correlation between ascending aorta dilatation and decreased SPA could be related to collagen re-synthesis [26].

Alterations in the vascular wall intimal extracellular matrix collagen turnover play a role in the development 
of atherosclerotic plaques, vascular remodeling, and plaque ruptures, causing acute coronary syndrome [2729]. This is further supported by intravascular ultrasound studies on SCF patients with prominent intimal hyperplasia and extensive calcification [3, 30]. Furthermore, a study of 321 patients revealed that SPA was related to coronary artery disease and other diffuse clinical characteristics [12].

Our results showed that SPA can be used as a predictor of SCF. This was the first study to employ a serum prolidase cut-off value for prediction of SCF; a serum prolidase level of $693.3 \mathrm{U} / \mathrm{I}$ was found to be predictive of SCF. Increased SPA may indicate an increased vascular intimal collagen turnover rate in SCF patients; therefore, endothelial dysfunction can lead to increased microvascular resistance, and may play a pivotal role in the pathophysiology of SCF. Serum prolidase activity can be measured easily, and in adults does not vary greatly. Therefore, it may be an appropriate measure for use in managing SCF. However, larger studies are needed to confirm our hypothesis.

The limitations of this study included the small number of patients, the cross-sectional design, and the lack of follow-up of the patients. Because of the lack of longterm follow-up data, conclusions regarding the predictive value of SPA for cardiovascular events could not be reached. Measuring serum and urine proline or hydroxyproline levels would add to the value of this study; however, we did not have the opportunity to perform these measurements.

\section{Conclusions}

This study is the first report of a correlation between SPA and SCF, and suggests that increased prolidase activity may play a role in the development of SCF. Although the specificity of SPA is low, it may be used in the management and follow-up of SCF patients. However, further clinical studies are needed to clarify the pathophysiological role of serum prolidase activity in SCF.

\section{Conflict of interest}

The authors declare no conflict of interest.

\section{References}

1. Tambe AA, Demany MA, Zimmerman HA, Mascarenhas E. Angina pectoris and slow flow velocity of dye in coronary arteries a new angiographic finding. Am Heart J 1972; 84: 66-71.

2. Kemp HG Jr, Vokonas PS, Cohn PF, Gorlin R. The anginal syndrome associated with normal coronary arteriograms. Report of a six year experience. Am J Med 1973; 54: 735-42.

3. Pekdemir H, Cin VG, Cicek D, et al. Slow coronary flow may be a sign of diffuse atherosclerosis. Contribution of FFR and IVUS. Acta Cardiol 2004; 59: 127-33.

4. Mosseri M, Yarom R, Gotsman MS, Hasin Y. Histologic evidence for small-vessel coronary artery disease in patients with angina pectoris and patent large coronary arteries. Circulation 1986; 7: 964-72.

5. Mangieri M, Macchiarelli G, Ciavolella M, et al. Slow coronary flow: clinical and histopathological features in patients with otherwise normal epicardial coronary arteries. Cathet Cardiovasc Diagn 1996; 37: 375-81.

6. Kurtoglu N, Akcay A, Dindar I. Usefulness of oral dipyridamole therapy for angiographic slow coronary artery flow. Am J Cardiol 2001; 87: 777-9.

7. Hein S, Schaper J. The extracellular matrix in normal and diseased myocardium. J Nucl Cardiol 2001; 8: 188-96.

8. Surazynski A, Miltyk W, Palka J, Phang JM. Prolidase-dependent regulation of collagen biosynthesis. Amino Acids 2008; 35: 731-8.

9. Oxlund $\mathrm{H}$, Andreassen TT. The roles of hyaluronic acid, collagen and elastin in the mechanical properties of connective tissues. J Anat 1980; 131: 611-20.

10. Palka JA, Phang JM. Prolidase activity in fibroblasts is regulated by interaction of extracellular matrix with cell surface integrin receptors. J Cell Biochem 1997; 67: 166-75.

11. Zanaboni G, Dyne KM, Rossi A, et al. Prolidase deficiency: biochemical study of erythrocyte and skin fibroblast prolidase activity in Italian patients. Haematologica 1994; 79: 13-8.

12. Yildiz A, Demirbag R, Yilmaz R, et al. The association of serum prolidase activity with the presence and severity of coronary artery disease. Coron Artery Dis 2008; 19: 319-25.

13. Rabus M, Demirbag R, Yildiz A, et al. Association of prolidase activity, oxidative parameters, and presence of atrial fibrillation in patients with mitral stenosis. Arch Med Res 2008; 39: 519-24.

14. Demirbag R, Yildiz A, Gur M, et al. Serum prolidase activity in patients with hypertension and its relation with left ventricular hypertrophy. Clin Biochem 2007; 40: 1020-5.

15. Myara I, Charpentier C, Lemonnier A. Optimal conditions for prolidase assay by proline colorimetric determination: application to iminodipeptiduria. Clin Chim Acta 1982; 125: 193-205.

16. Chinard FP. Photometric estimation of proline and ornithine. J Biol Chem 1952; 199: 91-5.

17. Gibson CM, Cannon CP, Daley WL, et al. TIMI frame count: a quantitative method of assessing coronary artery flow. Circulation 1996; 93: 879-88.

18. Kawakami K, Shimada T, Nakayama K, et al. Angina pectoris due to possible vasospasm of small coronary arteries. Clin Cardiol 1991; 14: 775-7.

19. Cannon RO 3rd, Epstein SE. "Microvascular angina" as a cause of chest pain with angiographically normal coronary arteries. Am J Cardiol 1988; 61: 1338-43.

20. Goel PK, Gupta SK, Agarwal A, Kapoor A. Slow coronary flow: a distinct angiographic subgroup in syndrome $X$. Angiology 2001; 52: 507-14.

21. Palka JA, Karna E, Miltyk W. Fibroblast chemotaxis and prolidase activity modulation by insulin-like growth factor II and mannose 6-phosphate. Mol Cell Biochem 1997; 168: 177-83.

22. Iyidogan YO, Gurdol F, Oner P. Evaluation of serum prolidase activity as bone turnover index. Tıp Fak Mecmuası 1999; 62: 301-6.

23. Erbagci AB, Araz M, Erbagci A, et al. Serum prolidase activity as a marker of osteoporosis in type 2 diabetes mellitus. Clin Biochem 2002; 35: 263-8.

24. Altindag O, Erel O, Aksoy N, et al. Increased oxidative stress and its relation with collagen metabolism in knee osteoarthritis. Rheumatol Int 2007; 27: 339-44. 
25. Gejyo F, Kishore BK, Arakawa M. Prolidase and prolinase activities in the erythrocytes of patients with chronic uremia. Nephron 1983; 35: 58-61.

26. Akcakoyun M, Pala S, Esen O, et al. Dilatation of the ascending aorta is associated with serum prolidase activity. Tohoku J Exp Med 2010; 220: 273-7.

27. Galis ZS, Sukhova GK, Lark MW, Libby P. Increased expression of matrix metalloproteinases and matrix degrading activity in vulnerable regions of human atherosclerotic plaques. J Clin Invest 1994; 94: 2493-503.

28. Zeng B, Prasan A, Fung KC, et al. Elevated circulating levels of matrix metalloproteinase- 9 and -2 in patients with symptomatic coronary artery disease. Intern Med J 2005; 35: 331-5.

29. Thomas CV, Coker ML, Zellner JL, et al. Increased matrix metalloproteinase activity and selective upregulation in LV myocardium from patients with end-stage dilated cardiomyopathy. Circulation 1998; 97: 1708-15.

30. Yaymacı B, Dagdelen S, Demirkol O, Dindar I. Anatomic and functional differences between slow coronary flow and normal coronary flow. Study of IVUS. TGKD 2001; 5: 94-9. 